\title{
Telomere length and telomerase activity in osteoporosis and osteoarthritis (Review)
}

\author{
PERSEFONI FRAGKIADAKI ${ }^{1,2}$, DRAGANA NIKITOVIC ${ }^{3}$, KATERINA KALLIANTASI ${ }^{1}$, \\ EVANGELIA SARANDI ${ }^{1,4}$, MARIA THANASOULA ${ }^{4}$, POLYCHRONIS D. STIVAKTAKIS ${ }^{1,2}$, CHARITINI NEPKA $^{5}$, \\ DEMETRIOS A. SPANDIDOS ${ }^{6}$, THEODOROS TOSOUNIDIS ${ }^{7}$ and ARISTIDIS TSATSAKIS ${ }^{1,2}$ \\ ${ }^{1}$ Laboratory of Toxicology, Medical School, University of Crete, 71003 Heraklion; ${ }^{2}$ Spin-Off Toxplus S.A., \\ 71601 Heraklion; ${ }^{3}$ Laboratory of Anatomy-Histology-Embryology, Medical School, University of Crete, \\ 71003 Heraklion; ${ }^{4}$ Metabolomic Medicine, Health Clinic for Autoimmune and Chronic Diseases, 10674 Athens; \\ ${ }^{5}$ Department of Cytopathology, University Hospital of Larissa, 41110 Larissa; ${ }^{6}$ Laboratory of Clinical Virology; \\ ${ }^{7}$ Department of Orthopedics, School of Medicine, University of Crete, 71003 Heraklion, Greece
}

Received November 1, 2019; Accepted December 23, 2019

DOI: $10.3892 /$ etm.2019.8370

\begin{abstract}
Osteoarthritis (OA) and osteoporosis (OP) are associated skeletal pathologies and have as a distinct feature the abnormal reconstruction of the subchondral bone. OA and OP have been characterized as age-related diseases and have been associated with telomere shortening and altered telomerase activity (TA). This review discusses the role of telomeres and telomerase in OA and OP pathologies and focuses on the usability of telomere length (TL) and the rate of telomere shortening as potential disease biomarkers. A number of studies have demonstrated that telomere shortening may contribute to OA and OP as an epigenetic factor. Therefore, it has been claimed that the measurement of TL of chondrocytes and/or peripheral blood cells may be an appropriate marker for the evaluation of the progression of these diseases. However, there is a need to be perform further studies with larger cohorts, with the aim of obtaining objective results and a better understanding of the association between TL, inflammation and aging, in order to provide further insight into the pathophysiology of degenerative joint diseases.
\end{abstract}

Correspondence to: Professor Aristidis Tsatsakis, Laboratory of Toxicology, Medical School, University of Crete, Voutes, 71003 Heraklion, Greece

E-mail: tsatsaka@uoc.gr

Abbreviations: OA, osteoarthritis; OP, osteoporosis; BMD, bone mineral density; NSAIDs, non-steroidal anti-inflammatory drugs; ROS, reactive oxygen species; ECM, extracellular matrix; TL, telomere length; TERT, telomerase reverse transcriptase; TERC, telomerase RNA component; CAD, cardiovascular disease; TRF, telomere restriction fragment; RTL, leukocyte relative telomere length; MSCs, mesenchymal stromal cells; BM, bone marrow; PBL, peripheral blood length

Key words: osteoporosis, osteoarthritis, telomere length, telomerase activity, shortenings

\section{Contents}

1. Introduction

2. Aging in $\mathrm{OP}$ and $\mathrm{OA}$

3. Role of telomeres and telomerase in aging

4. Association between telomere length, OP and OA

5. Therapeutic implications

6. Conclusions and future perspectives

\section{Introduction}

Osteoarthritis (OA) is a joint disease characterized by the degeneration of articular cartilage and modifications in subchondral bone. Importantly, OA has a complex pathophysiology and its presentation is associated with pathologies of manifold joint tissues. Primary OA is usually a result of the interaction of genetic and epigenetic factors that remain to be fully defined $(1,2)$. However, joint inflammation, obesity, hormonal imbalance and a low calcium concentration are strongly associated with secondary OA $(3,4)$. Importantly, OA and osteoporosis (OP) are two skeletal pathologies which are closely associated and have as a distinct feature the abnormal reconstruction of subchondral bone (5).

OP is one of the most common diseases affecting elderly individuals worldwide (6). It is characterized by reduced bone mineral density (BMD) and the microarchitectural deterioration of bone tissue. Based on its etiology, OP is categorized into two distinct types, namely type I (postmenopausal) and type II (senile) (7,8). In type I OP, the pathology generally develops with the estrogen reduction following the onset of menopause which causes bone loss. During the progression of type I disease, pathological changes of the trabecular bone are the most common. On the other hand, type II OP, which usually occurs after the age of 70 , involves the thinning of both trabecular and cortical bone $(7,8)$. Factors associated with the presentation of OP include the absence of physical exercise, malnutrition, poor protein synthesis and the lack of vitamin $\mathrm{C}$, as well as low menopausal 
and postmenopausal estrogen secretion (6). Currently, various pharmacological options are available for the treatment of OP; however, in OA, the management of patients is mostly limited to pain reduction and diverse modifications of lifestyle (9). Patients with advanced OA may receive non-steroidal anti-inflammatory drugs (NSAIDs), undergo physical therapy or occupational therapy, as well as surgical procedures, including cortisone and lubrication injections. At the final stages of the disease, total joint replacement or osteotomy are common and are usually the only treatment options (10).

\section{Aging in OP and OA}

Even though OP and OA are clinically distinct pathologies, they have many similarities as regards the hallmarks of aging. Thus, both pathologies have been characterized as age-related diseases, determining the lifestyle of affected patients more challenging as compared to a healthy elderly population (6). In particular, OA decreases the mobility, productivity and quality of life of individuals and leads to an increase in morbidity, as well as the use of medication and social welfare expenditures that contribute to a substantial socioeconomic burden (8).

The key feature of $\mathrm{OA}$ is the presentation of senescent cartilage with different histopathological characteristics compared to the aging cartilage tissue that is normally present in a healthy elderly individual. In aging cartilage, chondrocytes exhibit a decreased number and a low ability to proliferate. Simultaneously, the synthesis and deposition of extracellular matrix (ECM) components, that plays an important role in bone homeostasis and pathophysiology (11), is diminished, resulting in the gradual thinning of the cartilage layers (12). Indeed, it has been demonstrated that alterations in the function of chondrocytes and the deposition of ECM components may weaken the structural properties of articular cartilage and render the joint susceptible to OA. Furthermore, the tissues of patients with OA exhibit clusters of chondrocytes at the site of the lesion with an altered metabolism and an increased ability to produce pro-inflammatory cytokines and matrix-degrading enzymes (12). Thus, the pathogenesis of OA is linked to aging through several mechanisms, including inflammation related to aging, obesity, senescence, oxidative stress, alterations in metabolism, as well as cell signaling due to epigenetic mechanisms. Importantly, chondrocyte senescence contributes to the decreased ability of chondrocytes to repair articular cartilage tissue (12-14).

OP is a chronic skeletal disease with a high frequency worldwide, which has as a main characteristic, the deteriorated bone microarchitecture associated with the co-presentation of low BMD. However, the pathology is asymptomatic, and is associated in the majority of cases with a high risk of bone fracture, resulting in significant morbidity and mortality. OP related to aging is the most common form of the disease. There are several pathways involved in the etiopathogenesis of OP, including metabolic, endocrine and mechanical factors, whereas chronic inflammation has also been shown to play an important role (15). Indeed, the well-established estrogen deficiency in postmenopausal women has been shown to enhance the release of inflammatory mediators, leading to postmenopausal OP. Moreover, the ability of estrogens to downregulate receptor activator of nuclear factor- $\kappa \mathrm{B}$ ligand
(RANKL) synthesis has been shown in osteoblasts and likewise in T- and B-cells (16). Indeed, it is the lack of estrogen that, through the enhancement in T-cell activities, induces the increased secretion of pro-osteoclastogenic cytokines and subsequent osteoclastogenesis (17). Concomitantly, immune cells have been recognized as factors contributing to the development of osteoporosis (18). Moreover, it has been suggested that chronic antigenic load and oxidative stress that accumulate with aging cause a low-grade inflammation associated with a decrease in bone formation, as well as in bone resorption. These processes together lead to an imbalance in bone remodeling and the increased prevalence of OP (18).

Oxidative stress is intimately associated with the mechanisms of aging and together with other aging-related factors, including inflammation, an altered metabolism, cellular senescence and mitochondrial dysfunction, induce gradual OA-dependent joint destruction and increased bone fragility in OP (19). Reactive oxygen species (ROS) are generated under physiological conditions during mitochondria respiratory chain activities or through the actions of oxidative enzymes. However, upon biological, mechanical or chemical stimulation, an imbalance between ROS formation and ROS elimination occurs, favoring oxidative damage. This imbalance leads to an uncontrolled ROS production that favors pro-oxidant processes and oxidative damage $(20,21)$. Importantly, previous studies have demonstrated that oxygen free radicals can directly cause DNA damage (22), particularly at the guanine-rich end parts of chromosomes termed telomeres, the association of which with OA, OP and aging is discussed below (23).

\section{Role of telomeres and telomerase in aging}

Telomeres are specific structures positioned at the end of chromosomes that together with specific protein complexes bound to them, provide DNA protection (24), ensuring genomic stability. Specifically, the repetitive 5'-TTAGGG-3' sequences of 70 to 100 nucleotides bound to telomeric interacting proteins provide a protective cap of the chromosomal DNA, resembling the end of a shoelace. Due to the inability of the enzyme DNA polymerase to preserve the length of the 3 ' overhang (the so-called 'end replication problem'), telomeres are deprived of a small number of nucleotides during each mitotic cycle, practically becoming shorter and shorter (25). Indeed, the 'telomere hypothesis of cellular aging' was postulated in 1992 by Harley et al (26), and the over the past few years, accumulating evidence has indicated that telomere length (TL), which can be affected by various lifestyle factors, is associated with aging and the onset of age-related diseases $(26,27)$. In particular, stem cell dysfunction caused by telomere shortening may be one of the mechanisms responsible for aging in both humans and mice (9). Of note, a recent study revealed that the administration of nutraceutical supplements to healthy individuals was implicated in TL maintenance (28).

Telomeres are replicated by a specialized ribonucleoprotein complex, known as telomerase, that consists of a protein component entitled telomerase reverse transcriptase (TERT) that serves as a catalytic subunit (29) and an essential telomerase RNA component (TERC or RT) (30). Importantly, the enzyme 
telomerase can reverse telomere shortening, as it contributes to sustaining TL. However, it exhibits a high activity only in a subgroup of highly proliferating adult somatic cells, e.g., stem and progenitor cells, activated lymphocytes, as well as germline cells (31), while in the majority of adult human somatic cells, the expression and activity of telomerase is undetectable.

Recently, however, Astragalus membranaceus root and its active component, cycloastragenol, has been shown to activate telomerase in human somatic cells, in vitro and in vivo (32). In agreement with this, de Jesus et al previously demonstrated that the TA-65 component from Astragalus membranaceus root induced the elongation of short telomeres and the neutralization of associated DNA damage, in a telomerase-dependent manner (33). In addition to these studies, it has recently been demonstrated that specific natural compounds can significantly activate telomerase in human peripheral blood mononuclear cells in vitro (28).

TL and telomerase activity are strongly associated with human health as they have been linked to several age-related diseases, such as cancer, cardiovascular disease (CAD), diabetes, rheumatoid arthritis and psychiatric disorders (34-38). Moreover, previous studies have suggested that female human fertility decreases with an increased maternal age and that various adverse factors, including reduced telomerase activity, can contribute to age-associated infertility in women $(39,40)$. Furthermore, Vakonaki et al recently demonstrated the existence of a link between TL and drug abuse, which ultimately results in premature biological aging (41).

Telomere shortening, which is the main cause of age-related diseases, can be perpetrated through two distinctive mechanisms (42). According to the first mechanism, telomeres physiologically shorten with each cell division, due to the end replication problem. Since cells have a pre-defined number of cell divisions, when they reach the stage of critically short telomeres, they become senescent (43). As regards the second mechanism, imbalanced ROS production and associated oxidative stress can cause DNA damage to the guanine residues of telomeres, inducing the erosion of single telomeres (44). In general, DNA damage at the site of telomeres caused by various environmental factors triggers a DNA-damage response that protects them from instability and shortening $(44,45)$. However, if this protective mechanism is dysfunctional, telomeres are exposed to several damaging agents, leading to their critical shortening.

From all the above, it is clear that the determination of TL and its maintenance through intervention, can highly contribute to the delay of the aging process and the treatment of several age-related diseases, leading to longevity. In that context, a distinction between short telomeres and critically short telomeres has been achieved, considering the pivotal role that the critically short telomeres play in cell homeostasis. Based on these findings and developments, the 'BIOTEL' database was recently created, that is able to calculate a wide range of TL statistics, biological age and applications telomere biology research (46). The utilization of BIOTEL and similar tools will facilitate the analysis and assessment of telomere biology data and their application in health care.

The current review focuses on the role of telomeres in OA and OP pathologies and discusses the usability of TL and the rate of telomere shortening as potential disease biomarkers.

\section{Association between telomere length, OP and OA}

In an early, milestone study, Oreffo et al demonstrated that the number of osteoblast progenitors in the bone marrow (BM) of patients with OP, compared to that of age-matched controls, was decreased (47). In women, this has been partly attributed to age-related alterations in hormone levels, regarding sex hormones. In the context of elucidating the association between the aging of BM-mesenchymal cells and the development of OP, telomere shortening has gained increasing attention. Previous studies have demonstrated that the proliferative and osteogenic capacity of cultured mesenchymal stromal cells (MSCs) isolated from patients with OP was significantly decreased, which can be a possible marker of premature aging (48). It has been suggested that the decreased proliferative ability of MSCs may be due to the overexpression of osteogenic inhibitors in these cells in the case of OP $(49,50)$. More specifically, differences have been observed between the mesenchymal cell transcriptomes in OP and non-OP aging populations, which may be due to epigenetic changes reflecting a specific OP-associated aging process $(49,50)$. These findings are further supported by in vivo mouse models, which demonstrated that the proliferation-independent dysfunction of telomeres can induce an attenuation of osteoblast differentiation in mice with accelerated aging (51).

However, even though various studies had examined TL and its association with the pathology of OP, there is a great deal of inconsistency among them. For instance, in a large cohort of unselected women, the blood leukocyte TL was shown to be associated with BMD, whereas clinical OP was associated with shorter telomeres (52). Another study estimated the TL of female patients with OP using the telomere restriction fragment (TRF) approach, which revealed a stable decrease in TL among the different age groups of patients. Moreover, telomere shortening in leukocytes was associated with BMD or bone loss, but only after correcting for age, where TL was found to be associated with longitudinal bone loss, regarding sites in the distal forearm region (53). On the other hand, an early study comparing TRFs from peripheral blood length (PBL) DNA from female patients with OP and age-matched controls did not find any significant alterations (54). Likewise, in a separate study, even though age was found to be associated with both TL and BMD, no significant association was observed between TL and BMD. These inconsistencies among different studies could be explained by the results of a recent study that was performed in a cohort of elderly Chinese female and male patients with OP. According to that study, in the case of the female patients, age affected the association of TL with BMD and OP, but not in the case of the male patients, strongly suggesting that the TL predictive role may be sex-specific (55).

In addition to the above, accumulating evidence demonstrates that HIV is a significant risk factor for a low BMD and fractures due to bone fragility (56). Recently, it was determined that in a cohort of women with HIV, there was an association between premature spinal bone loss and a shorter TL. In summary, further focused studies are required to evaluate the association of TL and OP, as well as the feasibility of utilizing TL as an OP prognostic index.

As regards OA and its association with TL, patients with OA also appear to acquire several abnormalities indicative of 
Table I. Association of TL with the progression of OA and OP pathologies.

\begin{tabular}{|c|c|c|c|}
\hline Authors/(Refs.), year & Sample demographics & Main endpoint & TL assay type \\
\hline Price et al (61), 2002 & $\begin{array}{l}\text { OA hip patients }(n=15) \text { vs. OA } \\
\text { knee patients }(n=30) \text { vs. healthy }(n=11)\end{array}$ & Shorter TL in OA patients & Southern blot analysis \\
\hline $\begin{array}{l}\text { Zhai et al (62), 2006; } \\
\text { Li et al (63), } 2012\end{array}$ & $\begin{array}{l}\text { OA hand patients }(n=160) \text { vs. healthy } \\
(n=926)\end{array}$ & Shorter TL in OA patients & Southern blot analysis \\
\hline Valdes et al (52), 2007 & $\begin{array}{l}\text { OP and BMD in females }(n=2,150) \\
\text { aged } 18-79 \text { years }\end{array}$ & $\begin{array}{l}\text { Shorter leukocyte TL is not } \\
\text { associated with decreased } \\
\text { BMD or OP }\end{array}$ & TRF \\
\hline Sanders et al (71), 2009 & $\begin{array}{l}\text { Individuals }(n=2,750) \\
\text { aged } 70-79 \text { years with OP or fractures }\end{array}$ & $\begin{array}{l}\text { TL is not associated with BMD, } \\
\text { OP, or fractures in older } \\
\text { men or women }\end{array}$ & qPCR \\
\hline Tang et al (72), 2010 & $\begin{array}{l}\text { BMD in hip in elderly individuals } \\
(\mathrm{n}=1,876)\end{array}$ & $\begin{array}{l}\text { TL was not associated with } \\
\text { either baseline BMD or bone loss } \\
\text { over a period of } 4 \text { years }\end{array}$ & qPCR \\
\hline Tamayo et al (64), 2010 & $\begin{array}{l}\text { OA }(n=34) \text { and OP }(n=35) \text { vs. healthy } \\
\text { individuals }(n=130)\end{array}$ & $\begin{array}{l}\text { No differences observed in OA, } \\
\text { but a decreased observed TL in OP }\end{array}$ & qPCR \\
\hline Tamayo et al (65), 2011 & $\begin{array}{l}\text { OA patients }(n=39) \text { and in healthy } \\
(n=20) \text { individuals in leukocytes and } \\
\text { in chondrocytes }\end{array}$ & $\begin{array}{l}\text { OA longer TL in chondrocytes } \\
\text { vs. leukocytes; no differences } \\
\text { between TL in chondrocytes and } \\
\text { leukocytes in healthy individuals }\end{array}$ & qPCR \\
\hline Nielsen et al (73), 2015 & $\begin{array}{l}\text { Samples of the lumbar spine (LS), } \\
\text { femoral neck (FN) and total hip (TH) } \\
\text { were evaluated in } 460 \text { healthy women }\end{array}$ & $\begin{array}{l}\text { TL and BMD were not associated, } \\
\text { but a shorter TL could predict } \\
\text { a lower BMD }\end{array}$ & qPCR \\
\hline Sibille et al (69), 2017 & $\begin{array}{l}\text { Women without OA but pain severity } \\
(\mathrm{n}=136)\end{array}$ & $\begin{array}{l}\text { Shorter TL women with } \\
\text { chronic pain severity }\end{array}$ & Southern blot analysis \\
\hline Poonpet et al (58), 2018 & $\begin{array}{l}\text { Patients with knee OA } \\
\text { vs. healthy controls }(n=140)\end{array}$ & $\begin{array}{l}\text { Negative associations of } \\
\text { angiogenetic cytokines with RTL }\end{array}$ & qPCR \\
\hline
\end{tabular}

OA, osteoarthritis; OP, osteoporosis; TL, telomere length; BMD, bone mineral density; TRF, telomere restriction fragment; RTL, leukocyte relative telomere length.

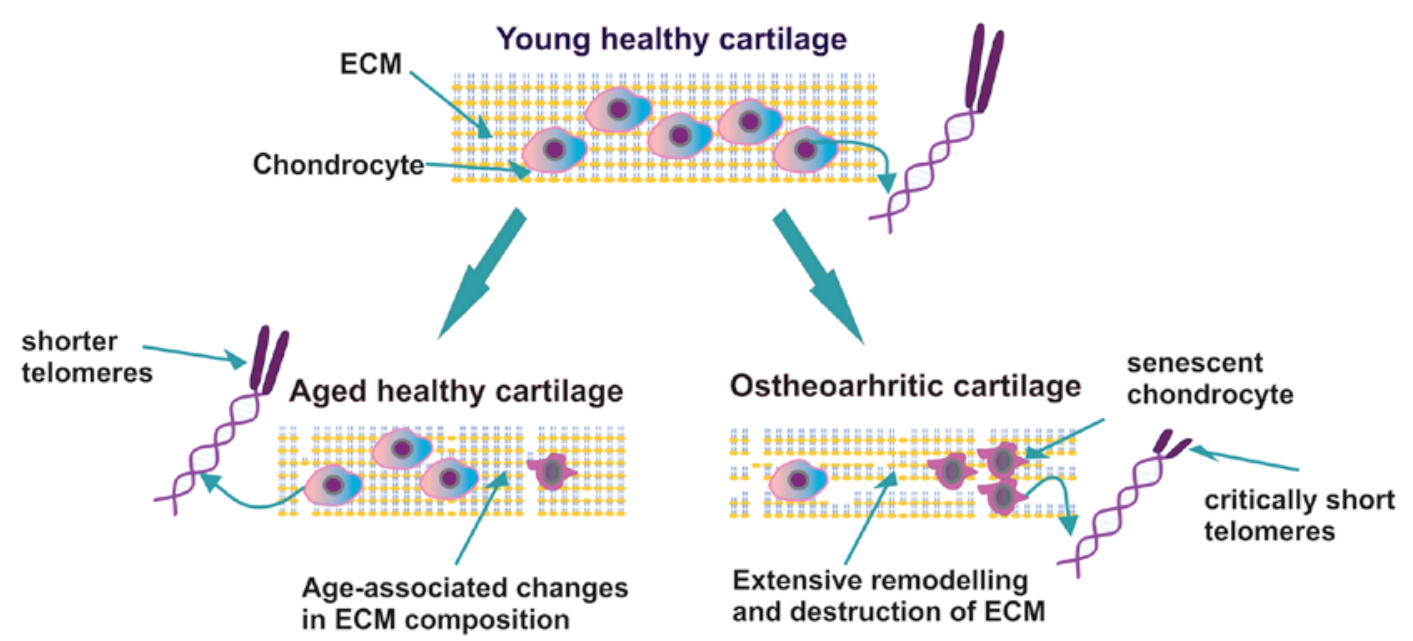

Figure 1. Aging is the main reason for the development of osteoarthritis and osteoporosis. ECM, extracellular matrix.

premature aging. Indeed, OA-affected chondrocytes tend to obtain a senescence-like phenotype (57), a fact that has initiated several attempts to evaluate the putative association of TL with the progression of OA. In that context, in a recent study, leukocyte relative telomere length (RTL) in patients with knee OA was compared to that of healthy controls (58). Additionally, possible associations between plasma angiogenic cytokine concentrations and leukocyte RTL were examined, indicating that TL was shorter in patients with knee OA compared to age-matched healthy controls. Notably, plasma hepatocyte growth factor (HGF), vascular endothelial growth factor (VEGF) and granulocyte-colony stimulating factor (G-CSF) 
levels were found to be negatively associated with leukocyte RTL. Therefore, that study indicated that high circulating angiogenic cytokine levels in the knees of patients with OA may reflect high oxidative stress and chronic inflammation, leading to subsequent telomere shortening (58). Indeed, overall oxidative stress can expedite telomere shortening either indirectly through increase in cell division, or directly acting on DNA telomere repeats, suggesting that redox balance is a prominent factor that regulates chondrocytes lifespan (59). In 2001, Martin and Buckwalter suggested that age-related changes in human cartilage chondrocytes may lead to cartilage erosion and osteoarthritis (60). Moreover, Price et al, utilizing Southern blot analysis, had demonstrated that patients with OA had shorter telomeres compared to unaffected chondrocytes in a group of 15 patients with hip OA, 30 patients with knee OA and a control group of 11 patients with no joint diseases (61). Additionally, Zhai et al measured relative TL in 160 patients with hand OA and in 926 patients without hand OA $(62,63)$. According to this study, the affected patients had shorter telomeres compared to the control group, suggesting that oxidative stress and inflammation within the affected joints led to telomere shortening due to accelerated DNA replication (61). In a separate study, Tamayo et al measured the average TL by qPCR in 34 patients with OA, in 35 patients with OP and 130 controls, and did not detect any differences between the patient and control groups (64). In 2011, these authors had estimated TL in human chondrocytes and peripheral blood leukocytes in 20 controls and 39 patients with knee and hip OA, and it was found that chondrocytes from patients with OA exhibited a significantly shorter TL when compared to chondrocytes from healthy individuals. Moreover, in patients with OA, telomeres were 1.6-fold longer in chondrocytes compared to leukocytes, indicating the existence of a cell-type specificity regarding TL. This hypotheses was corroborated by data from control subjects, where telomeres in chondrocytes were found to be even twice as long as telomeres from leukocytes (65). On the other hand, no difference was detected between the leukocytes and chondrocytes of controls, suggesting that the shorter telomeres in leukocytes may result from higher frequency of divisions of leukocytes compared to chondrocytes. Further supporting this theory, articular cartilage is a post-mitotic tissue, indicating that chondrocytes do not replicate often and in addition, according to a PCR-based assay decreased peripheral blood TL is markedly associated with the presentation of hand OA $(66,67)$.

Importantly, the shortening of chondrocyte telomeres caused by oxidative stress is a common aging-related process strongly associated with the incidence of cellular senescence, a finding that may have vast clinical importance in the early diagnosis and prognosis of OA. Harbo et al estimated the mean $\mathrm{TL}$, the number of short telomeres $(<1,500 \mathrm{bp})$ and the sites with senescence relative to the distance of the OA lesion, and concluded that all examined markers were highly associated with the distance from the lesion site (68). Importantly, the short telomere load was found to be a more significant marker for OA presentation, compared to the mean TL (68). Even though the respective study included only 3 patients with OA, it provides evidence that short telomere load of chondrocytes could be an important marker for OA diagnosis and prognosis. Based on these results, further studies focusing on the measurement of short telomere load in a larger dataset are warranted.
Notably, Sibille et al suggested that OA-associated pain was a severe stress factor that could affect TL. They measured TL in 136 women, aged between 45 and 85 years with or without symptomatic OA and categorized the participants into 5 groups according to the pain severity. This approach revealed that patients with chronic severe pain had shorter telomeres, although long TL did not correspond to low pain severity in this cohort (69).

Therefore, aging, OA and OP are independent processes; however, age is an important factor contributing to the progression of OA (4). Importantly, Ganguly et al among others, suggested that a decline in the number and 'fitness' of MSCs in the BM may be one of the main factors contributing to bone abnormalities in OP and OA (9) (Table I).

\section{Therapeutic implications}

To date, oxidative stress and chronic inflammation in patients with OA and OP are considered to be the main reasons leading to chondrocyte cellular senescence and apoptosis. Putatively, targeted antioxidant treatment protects chondrocytes and MSCs against oxidative stress-induced injury and associated inflammation. In that context, Hudita et al, using an in vitro scaffold-free 3-dimensional MSC culture model of chondrogenesis, demonstrated that acetylated fatty acids mixture from Celadrin reduced the secretion of inflammatory mediators and facilitated the chondrogenic differentiation process of human adipose-derived stem cells (70). Moreover, the estimation of TL in chondrocytes and/or $\mathrm{PBL}$ is a promising marker for the diagnosis and prognosis of OA.

\section{Conclusions and future perspectives}

OA and OP are two of the most common chronic diseases affecting the aging population with significant associated morbidity and mortality. The currently available therapies and disease progression markers do not meet the needs of these patients. Accumulating data indicate that telomere shortening may contribute to OA and OP as an epigenetic factor. Consequently, the measurement of TL of chondrocytes and/or PBL may prove to be appropriate markers for the evaluation of the progression of these diseases. It is important to identify the common mechanisms and etiologies among these pathologies and the aging process (Fig. 1). Indeed, options preventing the premature aging of mesenchymal cells could lead to novel therapies which specifically target altered bone formation in $\mathrm{OP}$ and OA. However, further studies with larger cohorts are required, in order to obtain objective results and to enhance our understanding of the association between TL, inflammation and aging. This may in turn provide further insight into the pathophysiology of degenerative joint diseases.

\section{Acknowledgements}

Not applicable.

\section{Funding}

This study was funded by Spin-Off Toxplus S.A. and supported by the Special Research Account of University of Crete (ELKE nos. 4602, 4920 and 3963). 


\section{Availability of data and materials}

Not applicable.

\section{Authors' contributions}

All the authors (PR, DN, KK, ES, MT, PDS, CN, DAS, TT and AT) contributed to the conception and design of the study. PF, $\mathrm{CN}$ and $\mathrm{KK}$ searched the literature for inclusion in the study that was then examined and reviewed by DN, ES and MT. PF and MT drafted and wrote the manuscript. AT and TT provided advice and critically revised the manuscript. All authors have read and approved the final version of the manuscript.

\section{Ethics approval and consent to participate}

Not applicable.

\section{Patient consent for publication}

Not applicable.

\section{Competing interests}

DAS is the Editor in Chief for the journal, but had no personal involvement in the reviewing process, or any influence in terms of adjudicating on the final decision, for this article. The other authors declare that they have no competing interests.

\section{References}

1. Panoutsopoulou K and Zeggini E: Advances in osteoarthritis genetics. J Med Genet 50: 715-724, 2013.

2. Yamasaki K, Nakasa T, Miyaki S, Ishikawa M, Deie M, Adachi N, Yasunaga $\mathrm{Y}$, Asahara $\mathrm{H}$ and Ochi M: Expression of MicroRNA-146a in osteoarthritis cartilage. Arthritis Rheum 60: 1035-1041, 2009.

3. Li H, Zeng C, Wei J, Yang T, Gao SG, Li YS, Luo W, Xiao WF, Xiong YL and Lei GH: Serum Calcium Concentration Is Inversely Associated With Radiographic Knee Osteoarthritis: A Cross-Sectional Study. Medicine (Baltimore) 95: e2838, 2016.

4. Roman-Blas JA, Castañeda S, Largo R and Herrero-Beaumont G Osteoarthritis associated with estrogen deficiency. Arthritis Res Ther 11: 241, 2009.

5. Dequeker J, Aerssens J and Luyten FP: Osteoarthritis and osteoporosis: Clinical and research evidence of inverse relationship. Aging Clin Exp Res 15: 426-439, 2003.

6. Franceschi C, Garagnani P, Morsiani C, Conte M, Santoro A, Grignolio A, Monti D, Capri M and Salvioli S: The continuum of aging and age-related diseases: common mechanisms but different rates. Front Med (Lausanne) 5: 61, 2018.

7. Cosman F, de Beur SJ, LeBoff MS, Lewiecki EM, Tanner B, Randall S and Lindsay R; National Osteoporosis Foundation: Clinician's Guide to Prevention and Treatment of Osteoporosis. Osteoporos Int 25: 2359-2381, 2014

8. Sözen T, Özışık L and Başaran NC: An overview and management of osteoporosis. Eur J Rheumatol 4: 46-56, 2017.

9. Ganguly P, El-Jawhari JJ, Giannoudis PV, Burska AN, Ponchel $\mathrm{F}$ and Jones EA: Age-related changes in bone marrow mesenchymal stromal cells: A potential impact on osteoporosis and osteoarthritis development. Cell Transplant 26: 1520-1529, 2017.

10. Nelson AE: Osteoarthritis year in review 2017: Clinical. Osteoarthritis Cartilage 26: 319-325, 2018.

11. Nikitovic D, Aggelidakis J, Young MF, Iozzo RV, Karamanos NK and Tzanakakis GN: The biology of small leucine-rich proteoglycans in bone pathophysiology. J Biol Chem 287: 33926-33933, 2012.

12. Lotz M and Loeser RF: Effects of aging on articular cartilage homeostasis. Bone 51: 241-248, 2012.
13. Zhang M, Theleman JL, Lygrisse KA and Wang J: Epigenetic mechanisms underlying the aging of articular cartilage and osteoarthritis. Gerontology 65: 387-396, 2019.

14. Martin JA and Buckwalter JA: Roles of articular cartilage aging and chondrocyte senescence in the pathogenesis of osteoarthritis. Iowa Orthop J 21: 1-7, 2001.

15. Ginaldi L, Di Benedetto MC and De Martinis M: Osteoporosis, inflammation and ageing. Immun Ageing 2: 14, 2005

16. Eghbali-Fatourechi G, Khosla S, Sanyal A, Boyle WJ, Lacey DL and Riggs BL: Role of RANK ligand in mediating increased bone resorption in early postmenopausal women. J Clin Invest 111: 1221-1230, 2003.

17. D'Amelio P, Grimaldi A, Di Bella S, Brianza SZM, Cristofaro MA, Tamone C, Giribaldi G, Ulliers D, Pescarmona GP and Isaia G: Estrogen deficiency increases osteoclastogenesis up-regulating T cells activity: A key mechanism in osteoporosis. Bone 43: 92-100, 2008.

18. Ponzetti M and Rucci N: Updates on Osteoimmunology: What's New on the Cross-Talk Between Bone and Immune System. Front Endocrinol 10: 236, 2019.

19. Wauquier F, Leotoing L, Coxam V, Guicheux J and Wittrant Y: Oxidative stress in bone remodelling and disease. Trends Mol Med 15: 468-477, 2009.

20. Sies H, Cadenas E, Symons MCR and Scott G: Oxidative stress: Damage to intact cells and organs. Philos Trans R Soc Lond B Biol Sci 311: 617-631, 1985.

21. Valko M, Leibfritz D, Moncol J, Cronin MT, Mazur M and Telser J: Free radicals and antioxidants in normal physiological functions and human disease. Int J Biochem Cell Biol 39: 44-84, 2007.

22. Fischer-Nielsen A, Corcoran GB, Poulsen HE, Kamendulis LM and Loft S: Menadione-induced DNA fragmentation without 8-oxo-2'-deoxyguanosine formation in isolated rat hepatocytes. Biochem Pharmacol 49: 1469-1474, 1995.

23. Yermilov V, Rubio J, Becchi M, Friesen MD, Pignatelli B and Ohshima H: Formation of 8-nitroguanine by the reaction of guanine with peroxynitrite in vitro. Carcinogenesis 16: 2045-2050, 1995.

24. Masutomi K, Possemato R, Wong JM, Currier JL, Tothova Z, Manola JB, Ganesan S, Lansdorp PM, Collins K and Hahn WC: The telomerase reverse transcriptase regulates chromatin state and DNA damage responses. Proc Natl Acad Sci USA 102: 8222-8227, 2005 .

25. Aubert $\mathrm{G}$ and Lansdorp PM: Telomeres and aging. Physiol Rev 88: 557-579, 2008

26. Harley CB, Vaziri H, Counter CM and Allsopp RC: The telomere hypothesis of cellular aging. Exp Gerontol 27: 375-382, 1992.

27. Saretzki G: Telomeres, Telomerase and Ageing. Subcell Biochem 90: 221-308, 2018

28. Tsoukalas D, Fragkiadaki P, Docea AO, Alegakis AK, Sarandi E, Thanasoula M, Spandidos DA, Tsatsakis A, Razgonova MP and Calina D: Discovery of potent telomerase activators: Unfolding new therapeutic and anti-aging perspectives. Mol Med Rep 20: 3701-3708, 2019.

29. Counter CM, Meyerson M, Eaton EN and Weinberg RA: The catalytic subunit of yeast telomerase. Proc Natl Acad Sci USA 94 9202-9207, 1997.

30. Blackburn EH: Switching and signaling at the telomere. Cell 106: 661-673, 2001

31. Kosebent EG, Uysal F and Ozturk S: Telomere length and telomerase activity during folliculogenesis in mammals. J Reprod Dev 64: 477-484, 2018.

32. Yu Y, Zhou L, Yang Y and Liu Y: Cycloastragenol: An exciting novel candidate for age-associated diseases. Exp Ther Med 16: 2175-2182, 2018.

33. Bernardes B de Jesus: Schneeberger K, Vera E, Tejera A, Harley CB and Blasco MA: The telomerase activator TA-65 elongates short telomeres and increases health span of adult/old mice without increasing cancer incidence. Aging Cell 10: 604-621, 2011.

34. Fujii H, Shao L, Colmegna I, Goronzy JJ and Weyand CM: Telomerase insufficiency in rheumatoid arthritis. Proc Natl Acad Sci USA 106: 4360-4365, 2009.

35. VakonakiE, Tsiminikaki K, Plaitis S, Fragkiadaki P, Tsoukalas D, Katsikantami I, Vaki G, Tzatzarakis MN, Spandidos DA and Tsatsakis AM: Common mental disorders and association with telomere length (Review). Biomed Rep 8: 111-116, 2018.

36. Wu KD, Orme LM, Shaughnessy J Jr, Jacobson J, Barlogie B and Moore MA: Telomerase and telomere length in multiple myeloma: Correlations with disease heterogeneity, cytogenetic status, and overall survival. Blood 101: 4982-4989, 2003. 
37. Willeit P, Willeit J, Brandstätter A, Ehrlenbach S, Mayr A, Gasperi A, Weger S, Oberhollenzer F, Reindl M, Kronenberg F, et al: Cellular aging reflected by leukocyte telomere length predicts advanced atherosclerosis and cardiovascular disease risk. Arterioscler Thromb Vasc Biol 30 1649-1656, 2010.

38. Calado RT and Young NS: Telomere diseases. N Engl J Med 361: 2353-2365, 2009.

39. Fragkiadaki P, Tsoukalas D, Fragkiadoulaki I, Psycharakis C, Nikitovic D, Spandidos DA and Tsatsakis AM: Telomerase activity in pregnancy complications (Review). Mol Med Rep 14: 16-21, 2016.

40. Vasilopoulos E, Fragkiadaki P, Kalliora C, Fragou D, Docea AO, Vakonaki E, Tsoukalas D, Calina D, Buga AM, Georgiadis $\mathrm{G}$, et al: The association of female and male infertility with telomere length (Review). Int J Mol Med 44: 375-389, 2019.

41. Vakonaki E, Tzatzarakis M, Tsiminikai K, Nathena D, Fragkiadaki P, Kalliantasi K, Kanaki K, Vaki G, Plaitis S, Tsoukalas D, et al: Effect of chronic and heavy drug abuse on biological aging. World Acad Sci J 1: 67-73, 2019.

42. Hemann MT, Strong MA, Hao LY and Greider CW: The shortest telomere, not average telomere length, is critical for cell viability and chromosome stability. Cell 107: 67-77, 2001.

43. Muraki K, Nyhan K, Han L and Murnane JP: Mechanisms of telomere loss and their consequences for chromosome instability. Front Oncol 2: 135, 2012

44. Thanasoula M, Escandell JM, Martinez P, Badie S, Muñoz P, Blasco MA and Tarsounas M: p53 prevents entry into mitosis with uncapped telomeres. Curr Biol 20: 521-526, 2010.

45. Thanasoula M, Escandell JM, Suwaki N and Tarsounas M: ATM/ATR checkpoint activation downregulates CDC25C to prevent mitotic entry with uncapped telomeres. EMBO J 31: 3398-3410, 2012

46. Tsatsakis A, Tsoukalas D, Fragkiadaki P, Vakonaki E, Tzatzarakis M, Sarandi E, Nikitovic D, Tsilimidos G and Alegakis AK: Developing BIOTEL: A Semi-Automated Spreadsheet for Estimating Telomere Length and Biological Age. Front Genet 10: 84, 2019.

47. Oreffo RO, Bennett A, Carr AJ and Triffitt JT: Patients with primary osteoarthritis show no change with ageing in the number of osteogenic precursors. Scand J Rheumatol 27: 415-424, 1998

48. Rodríguez JP, Garat S, Gajardo H, Pino AM and Seitz G: Abnormal osteogenesis in osteoporotic patients is reflected by altered mesenchymal stem cells dynamics. J Cell Biochem 75 414-423, 1999.

49. Benisch P, Schilling T, Klein-Hitpass L, Frey SP, Seefried L, Raaijmakers N, Krug $M$, Regensburger $M$, Zeck $S$ Schinke T, et al: The transcriptional profile of mesenchymal stem cell populations in primary osteoporosis is distinct and shows overexpression of osteogenic inhibitors. PLoS One 7: e45142, 2012.

50. Zhou Z, Gao M, Liu Q and Tao MD: Comprehensive transcriptome analysis of mesenchymal stem cells in elderly patients with osteoporosis. Aging Clin Exp Res 27: 595-601, 2015.

51. Wang H, Chen Q, Lee SH, Choi Y, Johnson FB and Pignolo RJ: Impairment of osteoblast differentiation due to proliferation-independent telomere dysfunction in mouse models of accelerated aging. Aging Cell 11: 704-713, 2012.

52. Valdes AM, Richards JB, Gardner JP, Swaminathan R, Kimura M, Xiaobin L, Aviv A and Spector TD: Telomere length in leukocytes correlates with bone mineral density and is shorter in women with osteoporosis. Osteoporos Int 18: 1203-1210, 2007.

53. Bekaert S, Van Pottelbergh I, De Meyer T, Zmierczak H, Kaufman JM, Van Oostveldt P and Goemaere S: Telomere length versus hormonal and bone mineral status in healthy elderly men. Mech Ageing Dev 126: 1115-1122, 2005.

54. Kveiborg M, Kassem M, Langdahl B, Eriksen EF, Clark BFC and Rattan SIS: Telomere shortening during aging of human osteoblasts in vitro and leukocytes in vivo: Lack of excessive telomere loss in osteoporotic patients. Mech Ageing Dev 106 261-271, 1999

55. Tao L, Huang Q, Yang R, Dai Y, Zeng Y, Li C, Li X, Zeng J and Wang Q: The age modification to leukocyte telomere length effect on bone mineral density and osteoporosis among Chinese elderly women. J Bone Miner Metab 37: 1004-1012, 2019.

56. Calmy A, Chevalley T, Delhumeau C, Toutous-Trellu L, Spycher-Elbes R, Ratib O, Zawadynski S and Rizzoli R: Long-term HIV infection and antiretroviral therapy are associated with bone microstructure alterations in premenopausal women. Osteoporos Int 24: 1843-1852, 2013.
57. McCulloch K, Litherland GJ and Rai TS: Cellular senescence in osteoarthritis pathology. Aging Cell 16: 210-218, 2017.

58. Poonpet T, Saetan N, Tanavalee A, Wilairatana V, Yuktanandana P and Honsawek S: Association between leukocyte telomere length and angiogenic cytokines in knee osteoarthritis. Int J Rheum Dis 21: 118-125, 2018.

59. Yudoh K, Nguyen vT, Nakamura H, Hongo-Masuko K, Kato T and Nishioka K: Potential involvement of oxidative stress in cartilage senescence and development of osteoarthritis: oxidative stress induces chondrocyte telomere instability and downregulation of chondrocyte function. Arthritis Res Ther 7: R380-R391, 2005

60. Martin JA and Buckwalter JA: Aging, articular cartilage chondrocyte senescence and osteoarthritis. Biogerontology 3 : 257-264, 2002.

61. Price JS, Waters JG, Darrah C, Pennington C, Edwards DR, Donell ST and Clark IM: The role of chondrocyte senescence in osteoarthritis. Aging Cell 1: 57-65, 2002.

62. Zhai G, Aviv A, Hunter DJ, Hart DJ, Gardner JP, Kimura M, Lu X, Valdes AM and Spector TD: Reduction of leucocyte telomere length in radiographic hand osteoarthritis: A population-based study. Ann Rheum Dis 65: 1444-1448, 2006.

63. Li J, Huang J, Dai L, Yu D, Chen Q, Zhang X and Dai K: miR-146a, an IL-1 $\beta$ responsive miRNA, induces vascular endothelial growth factor and chondrocyte apoptosis by targeting Smad4. Arthritis Res Ther 14: R75, 2012.

64. Tamayo M, Mosquera A, Rego JI, Fernández-Sueiro JL, Blanco FJ and Fernández JL: Differing patterns of peripheral blood leukocyte telomere length in rheumatologic diseases. Mutat Res 683: 68-73, 2010.

65. Tamayo M, Mosquera A, Rego I, Blanco FJ, Gosálvez J and Fernández JL: Decreased length of telomeric DNA sequences and increased numerical chromosome aberrations in human osteoarthritic chondrocytes. Mutat Res 708: 50-58, 2011.

66. Aigner T, Haag J, Martin J and Buckwalter J: Osteoarthritis: Aging of matrix and cells--going for a remedy. Curr Drug Targets 8: 325-331, 2007.

67. McAlindon T, Roberts M, Driban J, Schaefer L, Haugen IK, Smith SE, Duryea J, Cunha D, Blanco F, Fernández-Garcia JL, et al: Incident hand OA is strongly associated with reduced peripheral blood leukocyte telomere length. Osteoarthritis Cartilage 26: 1651-1657, 2018.

68. Harbo M, Delaisse JM, Kjaersgaard-Andersen P, Soerensen FB, Koelvraa S and Bendix L: The relationship between ultra-short telomeres, aging of articular cartilage and the development of human hip osteoarthritis. Mech Ageing Dev 134: 367-372, 2013.

69. Sibille KT, Chenc H, Bartley EJ, Riley J III, Gloverd TL, King CD, Zhang H, Cruz-Almeid Y, Goodin BR, Sotolongo A, et al: Accelerated aging in adults with knee osteoarthritis pain: consideration for frequency, intensity, time, and total pain sites. Pain Rep 2: e591, 2017

70. Hudita A, Galateanu B, Dinescu S, Costache M, Dinischiotu A, Negrei C, Stan M, Tsatsakis A, Nikitovic D, Lupuliasa D, et al: In Vitro Effects of Cetylated Fatty Acids Mixture from Celadrin on Chondrogenesis and Inflammation with Impact on Osteoarthritis. Cartilage 11: 88-97, 2020.

71. Sanders JL, Cauley JA, Boudreau RM, Zmuda JM, Strotmeyer ES, Opresko PL, Hsueh WC, Cawthon RM, Li R, Harris TB, et al; Health ABC Study: Leukocyte Telomere Length Is Not Associated With BMD, Osteoporosis, or Fracture in Older Adults: Results From the Health, Aging and Body Composition Study. J Bone Miner Res 24: 1531-1536, 2009.

72. Tang NL, Woo J, Suen EW, Liao CD, Leung JC and Leung PC: The effect of telomere length, a marker of biological aging, on bone mineral density in elderly population. Osteoporos Int 21: 89-97, 2010.

73. Nielsen BR, Linneberg A, Bendix L, Harboe M, Christensen K and Schwarz P: Association between leukocyte telomere length and bone mineral density in women 25-93 years of age. Exp Gerontol 66: 25-31, 2015 .

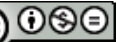

This work is licensed under a Creative Commons Attribution-NonCommercial-NoDerivatives 4.0 International (CC BY-NC-ND 4.0) License. 